\title{
Assessment of the Combined Effect of Epstein-Barr Virus and Plasmodium falciparum Infections on Endemic Burkitt Lymphoma Using a Multiplex Serological Approach
}

\section{OPEN ACCESS}

Edited by:

Magdalena Plebanski, Monash University, Australia

Reviewed by:

Katie Louise Flanagan, Monash University, Australia

Liisa Kaarina Selin,

University of Massachusetts Medical School, United States

*Correspondence: Carlota Dobaño carlota.dobano@isglobal.org

tShared authorship.

Specialty section: This article was submitted to Microbial Immunology,

a section of the journal

Frontiers in Immunology

Received: 24 July 2017 Accepted: 25 September 2017 Published: 26 October 2017

Citation:

Aguilar R, Casabonne D,

O'Callaghan-Gordo C, Vidal M, Campo JJ, Mutalima N, Angov E,

Dutta S, Gaur D, Chitnis CE, Chauhan V, Michel A, de Sanjosé S, Waterboer T, Kogevinas M, Newton $R$ and Dobaño C (2017) Assessment of the Combined Effect of Epstein-Barr Virus and Plasmodium falciparum Infections on Endemic Burkitt Lymphoma Using a Multiplex

Serological Approach.

Front. Immunol. 8:1284. doi: 10.3389/fimmu.2017.01284

\begin{abstract}
Ruth Aguilar ${ }^{1 \dagger}$, Delphine Casabonne ${ }^{2,3 t}$, Cristina O'Callaghan-Gordo $0^{2,4,5}$, Marta Vidal', Joseph J. Campo', Nora Mutalima ${ }^{6,7}$, Evelina Angov', Sheetij Dutta ${ }^{8}$, Deepak Gaur ${ }^{9,10}$, Chetan E. Chitnis ${ }^{9}$, Virander Chauhan ${ }^{9}$, Angelika Michel ${ }^{11}$, Silvia de Sanjosée ${ }^{2,3}$, Tim Waterboer ${ }^{11}$, Manolis Kogevinas $2,4,5,12$, Rob Newton ${ }^{13,14 t}$ and Carlota Dobaño ${ }^{1 * t}$
\end{abstract}

${ }^{1}$ ISGlobal, Barcelona Centre for International Health Research (CRESIB), Hospital Clínic, Universitat de Barcelona, Barcelona, Spain, ${ }^{2}$ CIBER Epidemiología y Salud Pública (CIBERESP), Madrid, Spain, ${ }^{3}$ Unit of Infections and Cancer, Cancer Epidemiology Research Programme, IDIBELL, Institut Català d'Oncologia, L'Hospitalet de Llobregat, Spain, ${ }^{4}$ ISGlobal, Center for Research in Environmental Epidemiology (CREAL), Barcelona, Spain, ${ }^{5}$ Universitat Pompeu Fabra (UPF), Barcelona, Spain, ${ }^{6}$ Department of Orthopaedic Surgery, Monash Health, Melbourne, VIC, Australia, ' Department of Surgery, School of Clinical Sciences, Monash University, Melbourne, VIC, Australia, ${ }^{8}$ WRAIR, Silver Spring, MD, United States, ${ }^{9}$ ICGEB, Delhi, India, ${ }^{10}$ School of Biotechnology, Jawaharlal Nehru University, New Delhi, India, ${ }^{11}$ German Cancer Research Center (DKFZ), Heidelberg, Germany, ${ }^{12}$ IMIM (Hospital del Mar Medical Research Institute), Barcelona, Spain, ${ }^{13}$ Epidemiology and Cancer Statistics Group, University of York, York, United Kingdom, ${ }^{14}$ MRC/UVRI Uganda Research Unit on AIDS, Entebbe, Uganda

Epstein-Barr virus (EBV) is a necessary cause of endemic Burkitt lymphoma (eBL), while the role of Plasmodium falciparum in eBL remains uncertain. This study aimed to generate new hypotheses on the interplay between both infections in the development of $\mathrm{eBL}$ by investigating the IgG and IgM profiles against several EBV and $P$. falciparum antigens. Serum samples collected in a childhood study in Malawi (2005-2006) from 442 HIV-seronegative children (271 eBL cases and 171 controls) between 1.4 and 15 years old were tested by quantitative suspension array technology against a newly developed multiplex panel combining 4 EBV antigens [Z Epstein-Barr replication activator protein (ZEBRA), early antigen-diffuse component (EA-D), EBV nuclear antigen 1, and viral capsid antigen p18 subunit (VCA-p18)] and 15 P. falciparum antigens selected for their immunogenicity, role in malaria pathogenesis, and presence in different parasite stages. Principal component analyses, multivariate logistic models, and elastic-net regressions were used. As expected, elevated levels of EBV IgG (especially against the lytic antigens ZEBRA, EA-D, and VCA-p18) were strongly associated with eBL [high vs low tertile odds ratio $(O R)=8.67,95 \%$ confidence interval $(C l)=4.81-15.64]$. Higher IgG responses to the merozoite surface protein 3 were observed in children with eBL compared with controls (OR $=1.29,95 \% \mathrm{Cl}=1.02-1.64)$, showing an additive interaction with EBV IgGs $(\mathrm{OR}=10.6,95 \% \mathrm{Cl}=5.1-22.2, P=0.05)$. Using elastic-net regression models, $\mathrm{eBL}$ serological profile was further characterized by lower lgM levels against $P$. falciparum preerythrocytic-stage antigen CelTOS and EBV lytic antigen VCA-p18 compared with controls. In a secondary analysis, abdominal Burkitt lymphoma had lower IgM to EBV 
and higher IgG to EA-D levels than cases with head involvement. Overall, this exploratory study confirmed the strong role of EBV in eBL and identified differential IgG and IgM patterns to erythrocytic vs preerythrocytic $P$. falciparum antigens that suggest a more persistent/chronic malaria exposure and a weaker lgM immune response in children with eBL compared with controls. Future studies should continue exploring how the malaria infection status and the immune response to $P$. falciparum interact with EBV infection in the development of eBL.

Keywords: endemic Burkitt lymphoma, Epstein-Barr virus, Plasmodium falciparum, IgG, IgM, multiplex, children, Africa

\section{INTRODUCTION}

Endemic Burkitt lymphoma (eBL), a highly aggressive B-cell non-Hodgkin lymphoma, is one of the most prevalent pediatric cancers in areas of sub-Saharan Africa where Plasmodium falciparum transmission is high. Epstein-Barr virus (EBV) is found in almost all cases of eBL and is a necessary agent in the development of the tumor (1). eBL peaks at age 6-7 years, is more frequent in males, and the most common sites of presentation are jaws and abdomen. The occurrence of eBL is limited to $P$. falciparum holoendemic areas (2), and there is strong evidence that the parasite reactivates EBV both in vivo and in vitro $(3,4)$.

P. falciparum-specific antigens cause polyclonal B-cell activation and increase EBV-infected B-cell survival (5). The parasite deregulates the activation-induced deaminase enzyme that is associated with somatic hypermutation and class switch recombination of immunoglobulin genes. This leads to DNA damage and increases the likelihood of c-myc translocations that, when happening in EBV-infected cells, leads to the development of lymphoma (6). In line with these observations, the International Agency for Research on Cancer categorized P. falciparum infection as a probable carcinogen (group 2A) in relation to the pathogenesis of eBL (7).

The intensity and timing of EBV and P. falciparum infections seem important features in eBL etiology. $P$. falciparum has been associated with earlier EBV acquisition and virus reactivation (8) and increases the frequency and duration of EBV events (9). Past and present exposure to infections in relation to eBL can be evaluated using serological markers. Four prior case-control studies in Uganda, Malawi, Kenya and Ghana measured P. falciparum antibodies to evaluate associations between malaria infection history and eBL (10-13). Those studies assessed IgGs against (i) schizont extract by indirect immunofluorescence assay (10) or ELISA (11), (ii) histidin rich protein II, circumsporozoite surface protein $\left(\mathrm{CSP}^{-\mathrm{NANP}_{6}}\right)$, SE36 subunit of serine repeat antigen-5 protein, and merozoite surface protein $1\left(\mathrm{MSP}_{-1}{ }_{42}\right)$ by ELISA (13), and (iii) MSP-1, liver stage antigen 1 (LSA-1), and apical membrane antigen 1 (AMA-1) (12) by quantitative suspension array technology (qSAT). Serological analyses based on few antigens in Uganda and Malawi suggested that EBV and malaria act jointly on eBL, but their combined effect was not evaluated in Kenya and Ghana.

$P$. falciparum is a complex parasite with 5,000-6,000 predicted proteins and with a life cycle that comprises morphologically, sexual and antigenically distinct stages (preerythrocytic and erythrocytic) that are targeted by stage-specific immunity. Some antigenic epitopes of immunity are known, but there is not yet a validated set of proteins that can accurately indicate recent or past malaria exposure. A larger panel of antigens than that used in past case-control eBL studies is desirable to better characterize malaria serological patterns. Furthermore, previous studies on eBL only looked at IgG responses to both infections, but no measurement of IgM, as marker of primoinfection/current infection, was taken into account.

Here, we determined IgG and IgM serological signatures using a new multiplex panel combining $4 \mathrm{EBV}$ and 15 P. falciparum antigens by qSAT in HIV-seronegative children from a cancer case-control study conducted in Malawi (11) to generate new hypotheses and get further insights into the interplay between EBV and P. falciparum infections in the development of eBL.

\section{MATERIALS AND METHODS}

\section{Study Participants}

Anonymized serum samples from 609 Malawian children were tested. Samples had been collected as part of a childhood malignancy and blood-borne virus study conducted at the Queen Elizabeth Hospital in Blantyre, Malawi, between 2005 and 2006 (11). Briefly, recruitment was performed in the pediatric oncology ward. The mother or guardian of each child was invited to participate in the study; written informed consent was provided, and standardized questionnaires administered. eBL was clinically diagnosed and in $75 \%$ of cases was confirmed by histology. HIV seropositives and infants $<1$ year who might have maternal IgGs were excluded from the current analysis. Overall, $271 \mathrm{eBL}$ cases and 171 controls between 1.4 and 15 years old were analyzed (Figure 1). Controls included 159 children diagnosed with Wilms tumor $(n=60)$, retinoblastoma $(n=11)$, rhabdomyosarcoma $(n=28)$, Kaposi sarcoma $(n=7)$, neuroblastoma $(n=13)$, hepatocellular carcinoma $(n=13)$, malignant teratoma $(n=3)$, bone tumor $(n=5)$, brain tumor $(n=3)$, ovarian tumor $(n=4)$, yolk sac tumor $(n=3)$, skin carcinoma $(n=2)$, salivary glands tumor $(n=1)$, metastasis $(n=1)$, and other soft tissue tumors $(n=5)$. Additional 12 controls were children admitted to the oncology ward with a provisional diagnosis of cancer, who were subsequently found to have non-malignant conditions. All children diagnosed with hematological cancers (including leukemia, non-Burkitt lymphoma, and small round blue cell tumors), Hodgkin lymphoma 


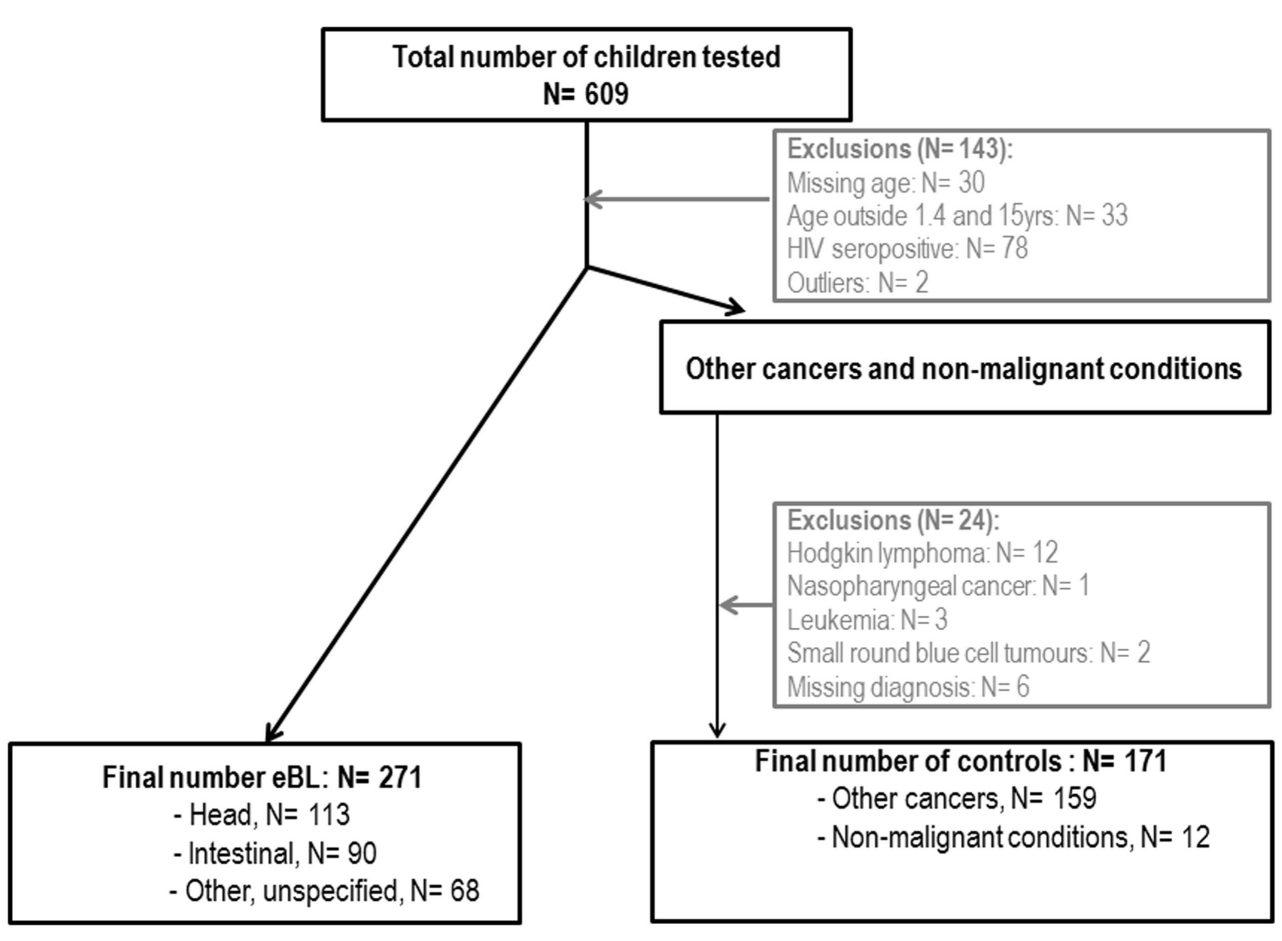

FIGURE 1 | Flowchart of endemic Burkitt lymphoma (eBL) cases and controls included in the study.

or nasopharyngeal cancer were excluded from the control group because of possible diagnostic overlap and/or previously known strong associations with EBV. Among eBL cases, 113 children presented with head tumors and 90 with abdominal tumors.

\section{Ethics Statement}

Ethical approval for the study was obtained from the Oxford Tropical Research Ethics Committee and the Malawian College of Medicine Research and Ethics Committee. The parent or guardian of each child provided written informed consent for their child to be included in the study in accordance with the Declaration of Helsinki.

\section{Laboratory Procedures EBV GST-Fusion Proteins}

Viral capsid antigen p18 subunit (VCA-p18), early antigen-diffuse component (EA-D), EBV nuclear antigen 1 (EBNA-1), and Z Epstein-Barr replication activator protein (ZEBRA) $(12,14)$ were expressed with pGEX vectors (Amersham) in Escherichia coli as double fusion proteins with N-terminal GST and a C-terminal peptide (tag). The proteins were affinity purified by incubation of glutathione displaying beads in bacterial lysate (15).

\section{P. falciparum Recombinant Proteins}

The antigens selected were based on their immunogenicity, their role in malaria pathogenesis and their presence in different parasite stages, some of them being vaccine candidates. Preerythrocytic antigens included the cell-traversal protein for ookinetes and sporozoites (CelTOS) (16), LSA-1 (17), sporozoite surface protein 2 (SSP2) also known as thrombospondinrelated adhesion protein (18), and CSP (19), supplied by Protein Potential (USA). Blood-stage antigens included AMA-1 3D7 (20), receptor-binding region $\mathrm{F} 2$ of the $175 \mathrm{kDa}$ erythrocyte binding antigen (F2) (EBA-175) (21), duffy binding-like (DBL) - $\alpha$ domain of PfEMP-1 (22), DBL3X domain of VAR2CSA PfEMP-1 (23), merozoite surface protein 3 (MSP-3) 3D7 (24), Plasmodium thrombospondin-related apical merozoite protein (PTRAMP) (25), reticulocyte binding-like homolog proteins 2 (Rh2) (26) and 5 (Rh5) (27), and cysteine-rich protective antigen 2 (CyRPA2) (28), produced at ICGEB. AMA-1 FVO (29) and MSP- $1_{42} 3 \mathrm{D} 7$ and FVO (30) were produced at WRAIR.

\section{Measurement of Antibodies to EBV and $P$. falciparum Proteins by qSAT}

A multiplex panel was established to quantify IgGs and IgMs against $P$. falciparum and EBV antigens using XMAPTM technology (Luminex Corp., Austin, TX, USA) (31). Distribution of eBL cases and controls was balanced through plates. Briefly, 1,000 microspheres were used per analyte and sample. EBV proteins were affinity purified on GSH-bearing beads, thus for EBV antigens, a fusion protein consisting of GST and tag without intervening viral antigen was coupled to beads to be used for background determination. P. falciparum proteins were covalently coupled directly to the beads and beads were blocked with BSA; thus for P. falciparum antigens, BSA coupled to the beads was used for background determination. Several plasma pools were used as positive controls in each plate: (i) a 
P. falciparum hyperimmune Mozambican adult volunteers' pool at 1/500 for IgG and 1/200 for IgM; (ii) an EBV immune Greek children pool (32) at 1/500 for IgG; and (iii) an EBV immune Malawi children pool (of samples with high IgM levels previously selected from the samples to be analyzed) at 1/200 for IgM. Several individual samples from malaria naïve European adults and non-EBV-exposed Greek children (32) were used as negative controls at $1 / 500$ for IgG and 1/200 for IgM. Study samples were tested in duplicates at $1 / 500$ and $1 / 20,000$ for IgG and $1 / 200$ and 1/20,000 for IgM. Two wells per plate were assayed without plasma added as blank controls. To block antibodies directed against residual bacterial proteins and GST present on the beads loaded with EBV proteins, plasmas were preincubated at a 1:20 dilution on a shaker at room temperature (RT) for $1 \mathrm{~h}$ in a serum preincubation buffer based on PBS, 1\% BSA, 0.05\% azide $\mathrm{pH} 7.4$ containing $2 \mathrm{~g} / \mathrm{L}$ lysate from bacteria expressing GST alone (15). After sample preincubation, beads were incubated with plasma samples for $1 \mathrm{~h}$. The plate was washed by pelleting microspheres using a magnet and resuspended with $0.05 \%$ Tween 20 -PBS. One hundred microliters of biotinylated antihuman IgG or IgM (Sigma, Tres Cantos, Spain) at 1/2,500 and 1/1,000, respectively, in PBS $1 \%$ BSA $0.05 \%$ azide pH 7.4 (assay buffer), were applied and incubated for $45 \mathrm{~min}$. The plate was washed as before, and $100 \mu \mathrm{L}$ of streptavidin-conjugated R-phycoerythrin (Invitrogen, Carlsbad, CA, USA) at 1/1,000 in assay buffer was added and incubated for $30 \mathrm{~min}$. All incubations were done at RT with plate agitation $(600 \mathrm{rpm})$ and protection from light. The plate was read using Luminex $200^{\mathrm{TM}}$ analyzer and Xponent software version 3.1, and at least 50 microspheres per analyte were acquired per sample. Median fluorescence intensity (MFI) with blank fluorescence subtracted was exported, and arbitrary units (AU) concentration for each antigen was calculated by extrapolation from an IgG or IgM standard curve prepared by coupling beads to commercially available purified antihuman IgG and antihuman IgM specific to the $\mathrm{F}\left(\mathrm{ab}^{\prime}\right)_{2}$ region, and performing the assay using purified human IgG and human IgM as primary antibody.

\section{Preprocessing of qSAT Data}

Standard curves were fitted using four or five-parameter logistic equations depending on the best fit. The sample dilution closer to the $\mathrm{EC}_{50}$ of the curve was used to calculate the concentration (AU) as: $\mathrm{EC}_{50}\left(\left(\left(E_{\min }-E_{\max }\right) /\left(\mathrm{MFI}-E_{\max }\right)\right) \wedge((1 / \text { Asym })-1)^{\wedge}(1 /\right.$ Hill $\left.)\right)$, where $\mathrm{EC}_{50}$ is the half maximal effective concentration, $E_{\min }$ is the minimum response, $E_{\max }$ is the maximum response, Asym is the asymmetry factor, and Hill is the slope. Final concentrations were corrected by the dilution factor.

Seropositivity per antigen was defined as AU above the mean +3 SDs of negative controls [non-malaria-exposed adults for P. falciparum and non-EBV-exposed children for EBV (32)]. EBV seropositivity was defined as positive IgGs against VCA-p18, EBNA-1 and EA-D, and positive IgM against VCA-p18 (33). $P$. falciparum seropositivity was defined as presence of antibodies to any of the parasite antigens.

\section{Statistical Analysis}

Raw Luminex data (AU) were log transformed and standardized to normalize and bring all of the variables into proportion with one another (with mean 0 and SD 1). All analyses were done primarily using continuous data to gain power and avoid loss of information (34). Correlations were examined by Pearson matrices. Associations between serological responses to each antigen and demographic factors were examined using linear regression models adjusted for age and sex, as appropriate. Principal component analysis (PCA) was performed with an orthogonal rotation on EBV and P. falciparum, separately, to obtain the structure of the data for each infection (Table S1 in Supplementary Material). Two and five components were identified for EBV and P. falciparum, respectively. Based on PCA loading results, the two derived EBV components were labeled as "IgM EBV pattern" and "IgG EBV pattern." Classical and multinomial logistic regression models were used to study the associations of antibodies, extracted principal components, and multiple seropositivity, with eBL. All models were adjusted for age (three categories: $\geq 1.41<3.75, \geq 3.75<7.64$, and $\geq 7.64$ years) and sex. Further adjustment was performed including "IgG EBV pattern" (as continuous).

Elastic-net regression models were used to build an antibody response signature associated with eBL accounting for the high correlation and high dimensional scenario. Tuning elastic-net parameters ( $\alpha$ and $\lambda$ ) were optimized using a 10-fold crossvalidation minimizing the deviance. The stability of the selected variables was assessed using bootstrap with replacement (number of iterations $N=500)$ with the pair of parameters $(\alpha, \lambda)$ optimized again at each bootstrap iteration. Variables were considered as highly important if their inclusion frequency was $\geq 70 \%$.

Both multiplicative and additive interactions (relative excess risk due to interaction RERI and synergy index S) were examined. As this is a hypothesis-generating study, we did not adjust for multiple comparisons, and results were interpreted for internal coherence and biological plausibility. Analyses were performed using STATA13 (35) and R (36) using the glmnet (37), c060 (38), and NMF packages (39).

\section{RESULTS}

\section{Demographic Characteristics of Study Participants}

Table 1 shows demographic characteristics of participants. Controls were younger than cases [mean age $( \pm S D): 6.3( \pm 3.7)$ and $7.8( \pm 2.9)$ years, respectively], with a proportion of children age of $<4$ years being much higher. Cases were more likely to be in the lower body mass index (BMI) tertile compared with the controls (51 vs $34 \%$, respectively). The majority of cases and controls came from the South of Malawi ( 96 vs $87 \%$, respectively). No differences were observed for other co-variables assessed.

\section{Serological Characteristics of Study Participants}

Overall, $88 \%$ of children were seropositive for EBV: $77 \%$ of controls and $96 \%$ of cases. Proportions by age group $(\geq 1.41<3.75$, $\geq 3.75<7.64$, and $\geq 7.64$ years) were 79,75 , and $77 \%$ among controls, and 88, 96, and 95\% among cases. There were no differences in EBV seropositivity by sex (86\% males vs $90 \%$ 
females). For P. falciparum, 99\% children were IgG seropositive (99\% cases and 98\% controls) and $84 \%$ IgM seropositive (85\% cases and $82 \%$ controls). Among controls, for most EBV

TABLE 1 | Basic demographic characteristics of study participants.

\begin{tabular}{|c|c|c|c|}
\hline & $\begin{array}{c}\text { Controls } \\
N(\%)\end{array}$ & $\begin{array}{l}\text { Cases } \\
N(\%)\end{array}$ & $P$-het \\
\hline & $N=171$ & $N=271$ & \\
\hline \multicolumn{4}{|l|}{ Age (years) } \\
\hline$\geq 1.41<3.75$ & 57 (33\%) & $16(6 \%)$ & \\
\hline$\geq 3.75<7.64$ & 57 (34\%) & $124(46 \%)$ & \\
\hline$\geq 7.64$ & 57 (33\%) & $131(48 \%)$ & $<0.0001$ \\
\hline Mean age (SD) & $6.3(3.7)$ & $7.8(2.9)$ & $<0.0001$ \\
\hline Range & $1.5-14.9$ & $1.4-14.6$ & \\
\hline \multicolumn{4}{|l|}{ Sex } \\
\hline Female & 75 (44\%) & $110(41 \%)$ & \\
\hline Male & $99(56 \%)$ & $161(59 \%)$ & 0.50 \\
\hline \multicolumn{4}{|l|}{ Body mass index $\left(\mathrm{kg} / \mathrm{m}^{2}\right)$} \\
\hline First tertile $(<14.18)$ & $54(34 \%)$ & $129(51 \%)$ & \\
\hline Second tertile $(\geq 14.18 \leq 15.97)$ & $53(33 \%)$ & 87 (34\%) & \\
\hline Third tertile $(\geq 15.97)$ & $53(33 \%)$ & $37(15 \%)$ & $<0.0001$ \\
\hline \multicolumn{4}{|l|}{ Country of residence } \\
\hline Malawi & $162(95 \%)$ & $254(94 \%)$ & \\
\hline Mozambique & $9(5 \%)$ & $16(6 \%)$ & 0.77 \\
\hline \multicolumn{4}{|l|}{ Region } \\
\hline Central & $17(10 \%)$ & $9(4 \%)$ & \\
\hline Northern & $4(2 \%)$ & $1(0 \%)$ & \\
\hline Southern & 142 (87\%) & $244(96 \%)$ & 0.003 \\
\hline \multicolumn{4}{|l|}{ Guardian at interview } \\
\hline Mother & $135(79 \%)$ & $204(76 \%)$ & \\
\hline Others & $36(21 \%)$ & $66(24 \%)$ & 0.41 \\
\hline
\end{tabular}

Numbers may not add to the total because of missing values.

$N$, number; P-het: P-heterogeneity.

The numbers in bold correspond to the associations that are statistically significant at the $5 \%$ level. and $P$. falciparum antigens, increasing age was associated with increasing antibody levels except for IgG VCA-p18 (Table S2 in Supplementary Material). Males tended to have lower IgM responses to several malaria antigens. No pattern was observed in the serological response by geographical region or by children's BMI.

\section{"IgG EBV Pattern" and IgG against $P$. falciparum MSP-3 Antigen Are Associated with eBL}

Strong correlations were observed between and within antibodies for both pathogens, especially between IgMs. Overall correlations tended to be stronger in controls than in cases (Figure 2). As expected, IgG to specific EBV antigens and the PCA component "IgG EBV pattern" were strongly positively associated with eBL \{adjusted odds ratio (OR) [95\% confidence interval $(\mathrm{CI})=3.73(2.75-5.08)]$; Figure 3$\}$. On the contrary, IgMs to EBV were not associated with eBL, except for IgMs against VCA-p18 that were negatively associated with eBL after adjusting for age and sex.

In relation to $P$. falciparum antigens, after controlling for age, sex, and "IgG EBV pattern," positive associations were observed for IgG against MSP-3 (Figure 3). In contrast to EBV results, none of the five PCA components derived from P. falciparum serology were associated with eBL after controlling for age, sex, and "IgG EBV pattern" (data not shown).

\section{IgGs against Multiple $P$. falciparum Erythrocytic Antigens Weakly Associated with eBL}

Overall, joint presence of antibodies to multiple $P$. falciparum antigens was not associated with eBL after adjustment for age, sex, and "IgG EBV pattern" (Table 2). When antibodies were

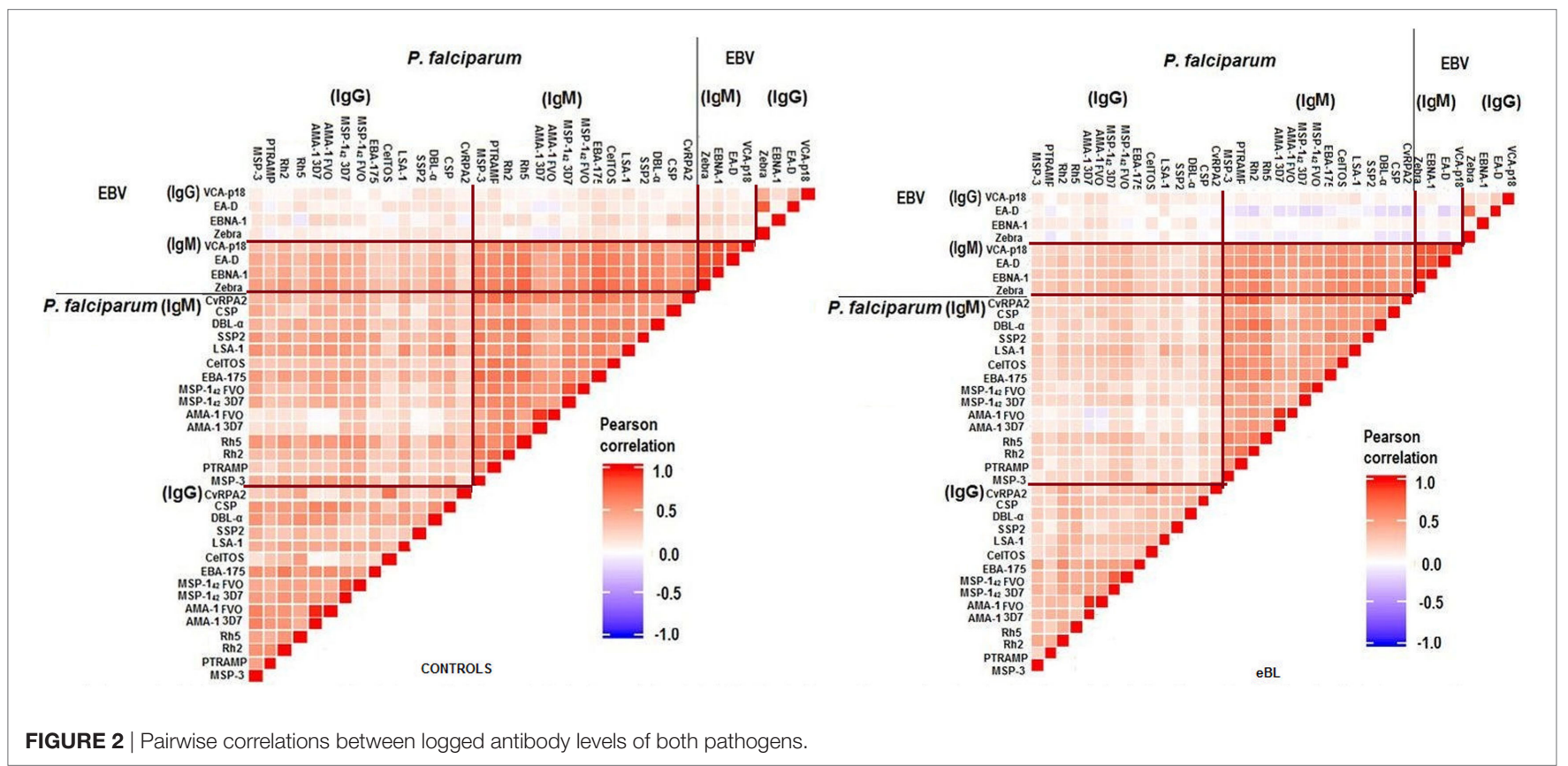




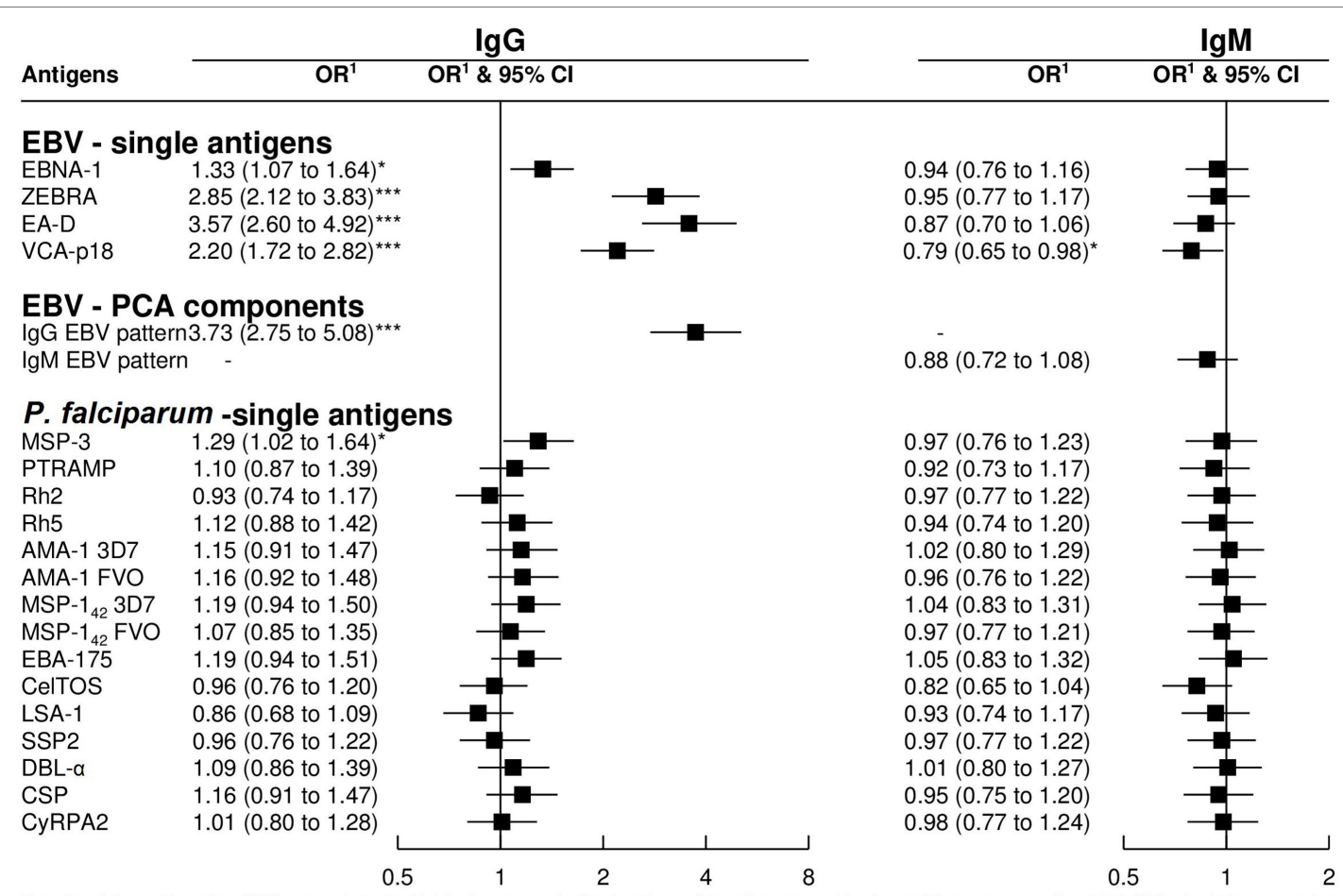

Results of the odds ratios (OR) were plotted with black squares indicating the point estimates and horizontal lines representing $95 \% \mathrm{Cl}$ for logistic regression models. 1: OR of Burkitt lymphoma for 1 standard deviation increase on log-transformed and standardized data for single antigens and standardized data for PCA components. Models examining the association with EBV antibodies are adjusted for age ( 3 groups) and sex, whereas those examining $P$. falciparum are adjusted for age ( 3 groups), sex and IgG EBV pattern. ${ }^{\star}: \mathrm{P}<0.05,{ }^{\star \star \star}: \mathrm{P}<0.0001$

FIGURE 3 | Odds ratios (ORs) for endemic Burkitt lymphoma in relation to lgG and lgM levels against single Epstein-Barr virus (EBV) and Plasmodium falciparum antigens and against EBV PCA components.

TABLE 2 | ORs of endemic Burkitt lymphoma for the burden of IgGs to Plasmodium falciparum antigens.

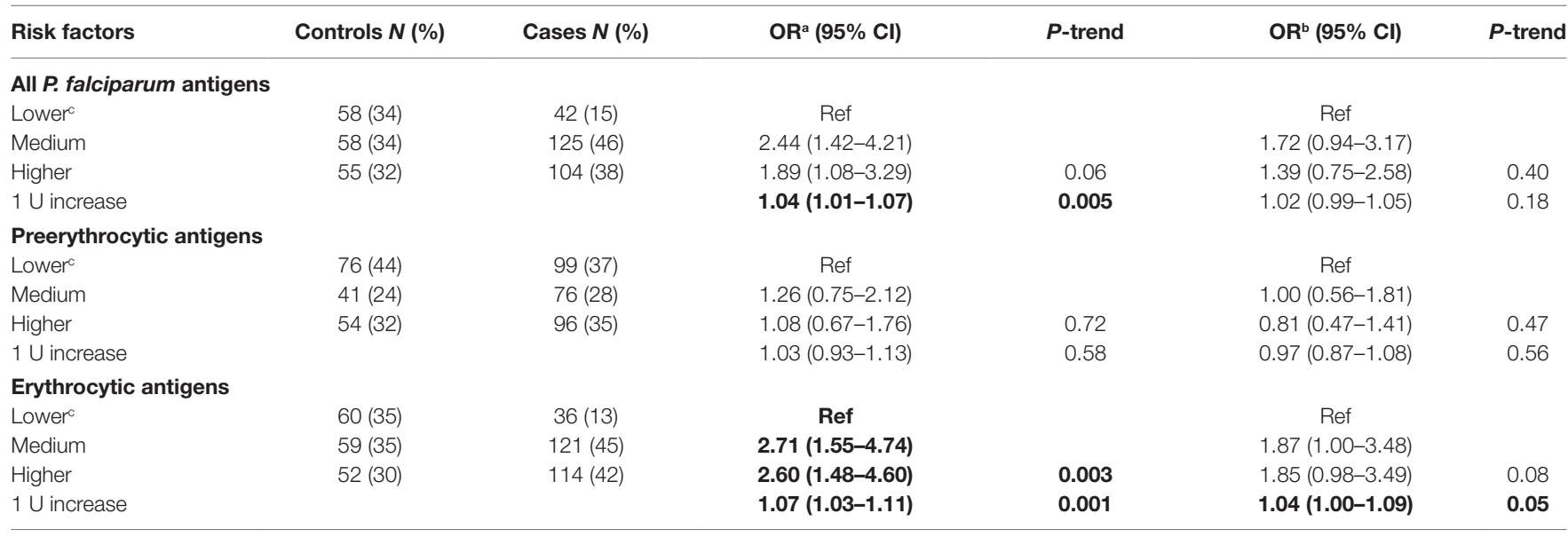

Ref, reference; $\mathrm{OR}$, odds ratio; $\mathrm{Cl}$, confidence interval.

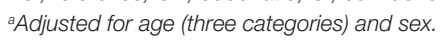

"Adjusted for age, sex, and the principal component analysis component "IgG Epstein-Barr virus pattern."

'The burden was calculated creating a score by adding tertiles values of the log-transformed data (coded as 1,2, and 3) for each antigen and dividing the score into three groups based on the control distribution. Preerythrocytic antigens: CelTOS, SSP2, CSP and LSA-1.

The numbers in bold correspond to the associations that are statistically significant at the $5 \%$ level.

further grouped following parasite stages, a positive association was observed for the joint presence of IgGs to erythrocytic antigens [OR $(95 \% \mathrm{CI})$ for seropositivity to third tertiles $=1.04$
(1.00-1.09)] but not for IgGs to preerythrocytic antigens. No associations were observed for joint presence of multiple IgMs (data not shown). 


\section{Antibody Response Signature for eBL}

The antibody response signature for eBL included antibodies to 7 antigens out of the 38 examined (Figure 4). Highest eBL positive ORs were observed for increasing IgGs against the three EBV lytic antigens EA-D, and VCA-p18 (OR = 1.53 and 1.29, respectively), and to a lesser extent for increasing IgG to the latent antigen EBNA-1 and the lytic antigen ZEBRA $(\mathrm{OR}=1.13$ for both antigens). Regarding the IgM EBV repertoire, OR of eBL decreased with increasing IgM to VCA-p18 $(\mathrm{OR}=0.97)$. In relation to Igs to $P$. falciparum, children with eBL were more likely to have higher IgG against MSP-3 (OR = 1.18) and lower IgM against CelTOS $(\mathrm{OR}=0.94)$.

\section{Additive Interaction between IgGs against EBV and MSP-3}

An additive effect of the "IgG EBV pattern" and IgG seropositivity to MSP-3 was observed that significantly increased the OR of eBL beyond the associated with each factor independently [OR (95\% CI) for high "IgG EBV pattern" and IgG seropositivity to MSP-3 compared with low/seronegativity = 10.6 (5.1-22.2)]. However, this interaction reached borderline statistical significance $(P=0.05)$ (Figure 5). No other interactions were observed.

\section{Association between Antibody Responses and Tumor Location}

Cases with abdominal tumors were older than cases with jaw tumors [mean (SD) age: $8.9(0.3)$ vs $6.9(0.2)$ years, respectively; $P<0.001$ ] and more likely to be female ( 47 vs $32 \%, P=0.03$ ). The association between tumor location and the assessed antibodies as well as PCA compounds was examined for both infections (Figure 6). Compared with controls, OR for abdominal tumors decreased with increasing IgM against EBV, whereas no difference was observed in children with head tumors (all $P$-values for heterogeneity between tumor location $<0.05$ ). Regarding IgGs to EBV antigens, only IgG to EA-D was positively associated with higher OR for abdominal tumors compared with head tumors $(P=0.05)$. No associations were observed for antibodies to $P$. falciparum and tumor location (data not shown).

\section{DISCUSSION}

This study aimed to give further insight into the combined effect of EBV and $P$. falciparum in the development of eBL by analyzing IgG and IgM patterns to $4 \mathrm{EBV}$ and $15 P$. falciparum antigens (10 never used before, including 3 preerythrocytic) in Malawian children from a cancer case-control study (11). As expected,

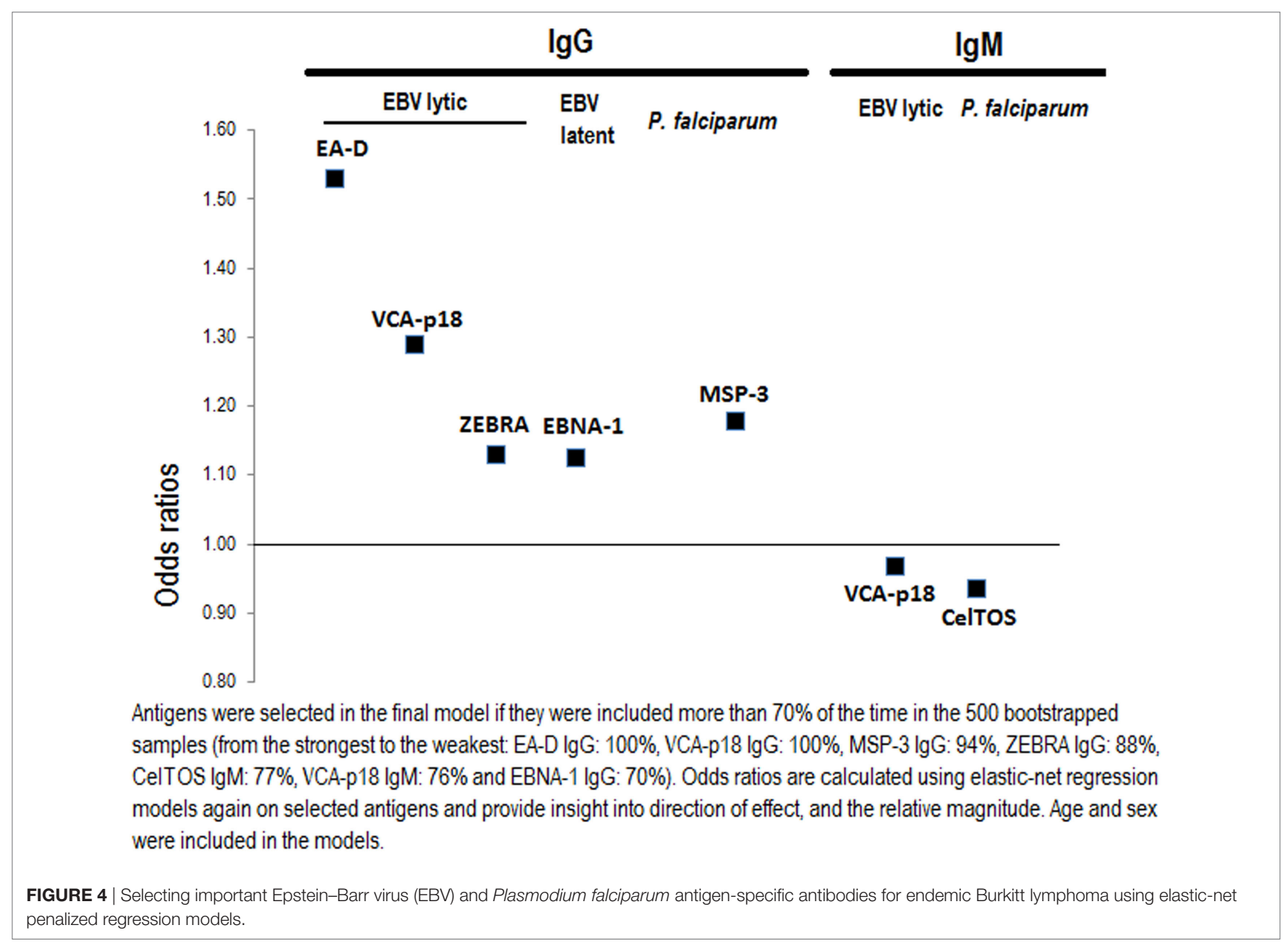




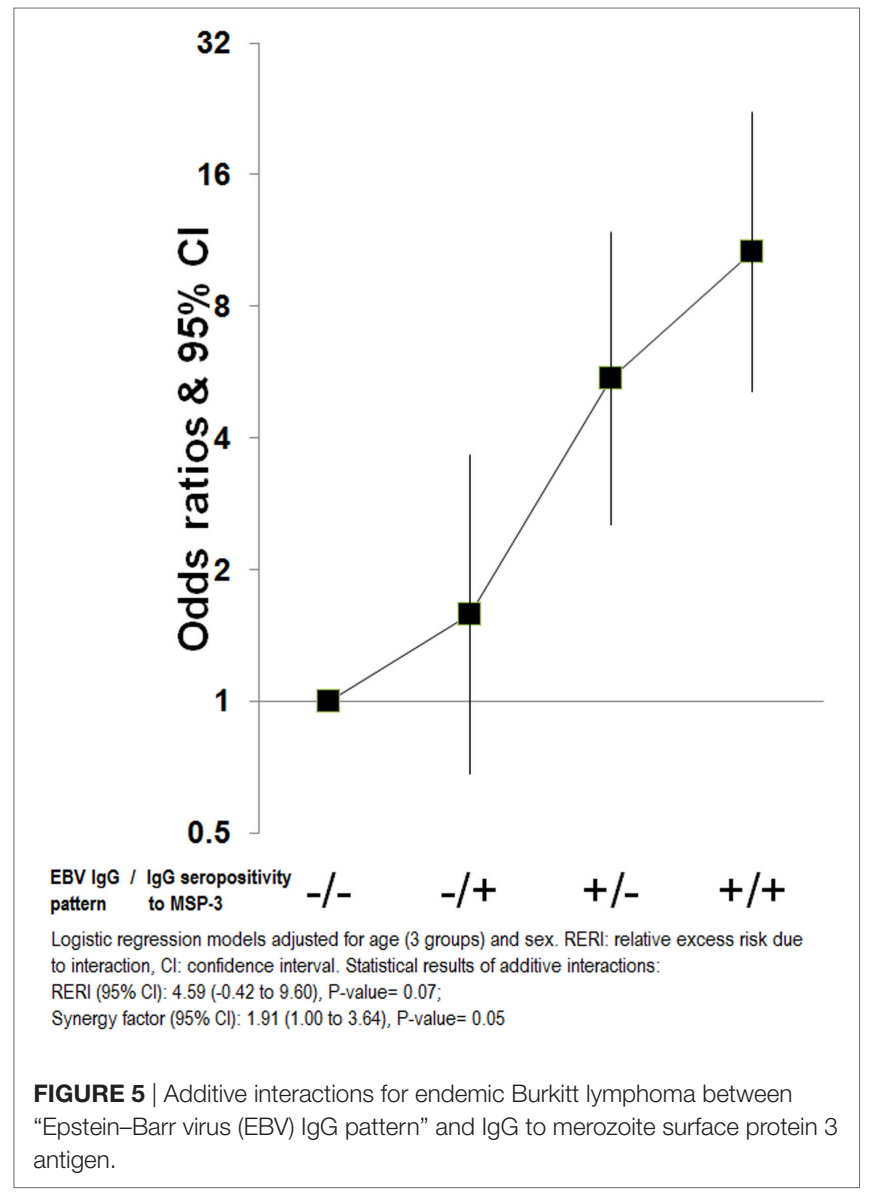

our data support a strong role of EBV in eBL, and a weak effect of malaria, but suggest that both infections might act jointly on $\mathrm{eBL}$, as shown by the additive interaction between elevated IgGs against EBV and MSP-3. In agreement with this observation, two recent studies have reported that the key determinants of EBV primary infection kinetics in children are the degree of malaria exposure and maternal antibody decay $(9,40)$.

The study population showed a high EBV seroconversion at a young age ( $>3 / 4$ of the controls age $\leq 3.8$ years), contrasting with recent estimates from Crete where only $50 \%$ of children at age 4 years had seroconverted (32). As expected, elevated EBV IgG levels were strongly positively associated with eBL $(10-12,41)$, whereas high anti-VCA-p18 IgM responses were negatively associated with eBL. In particular, IgG levels to the three EBV lytic antigens (EA-D, marker of active infection and viral reactivation, ZEBRA involved in the switch from latent to lytic stage, and VCAp18) were higher compared with IgG against EBNA-1 (latency marker). High IgM levels against VCA-p18 usually indicate recent primary EBV infection, although it may persist for months or might reappear in EBV reactivation. The negative association of high IgM anti-VCA-p18 levels with eBL suggests that newly EBV-infected children might be at lesser risk of eBL compared with children exposed for a longer time, or might indicate that in a malaria endemic region the long-term persistence of anti-VCA IgM responses may be associated with a better control of EBV infection (40).
In contrast to the strong EBV association, we only observed weak associations for few of the 15 antibodies measured against $P$. falciparum antigens, which is difficult to interpret as serological understanding of $P$. falciparum exposure and acquired immunity are not yet fully elucidated (42). However, the observed differences are probably related to the diverse immunogenicities of these antigens. The strongest and most consistent association was found for IgG against MSP-3, a highly immunogenic antigen involved in merozoite binding to human erythrocytes (43). Anti-MSP-3 IgG responses have been associated with protection against malaria in some sero-epidemiological studies $(44,45)$, but in others just showed to be exposure markers $(46,47)$. Higher burden of IgGs against multiple merozoite proteins was observed in eBL patients compared with controls suggesting that a persistent/ chronic malaria exposure might enhance EBV infection as well as reactivation. To deal with the issue of assessing associations for correlated EBV and P. falciparum antigen-specific antibodies, we used elastic-net regression models to select a subset of the most important antibodies for eBL, mutually adjusted for each other. While getting inference testing on the selected antigens for elastic-net is still an open statistical question, the results allow us to generate some new hypotheses. The higher IgM responses to the liver-stage antigen CelTOS were inversely associated with eBL, suggesting that newly malaria-infected children might be at lesser risk of eBL compared with children exposed for a longer time. Alternatively, the long-term persistence of anti-CelTOS IgM responses may be associated with a better control of malaria infection. CelTOS-specific cellular immunity has been detected among protected subjects in a controlled human malaria infection study (48), and antibodies to CelTOS have been shown to correlate with naturally acquired clinical immunity (49). Also, a recent study reports that anti-CelTOS responses prevent hepatocyte infection by sporozoites (50).

As secondary analysis, we observed that lower "EBV IgM pattern" and higher IgG responses to EA-D occurred in children with abdominal tumor presentation compared with head location. This differs to results from a previous study $(N=14$ jaw and 16 abdominal tumors) where higher anti-ZEBRA but not anti-EA-D IgG levels were associated with abdominal tumors (12). Here, IgG levels to EA-D highly correlated with ZEBRA IgG, particularly among cases, and consequently both IgG responses to ZEBRA and EA-D were elevated in children with abdominal vs head tumors, but results were only statistically significant for EA-D. We also observed that children with abdominal tumors were more likely to have lower EBV IgMs, suggesting that abdominal compared with jaw tumors may arise more frequently in long-standing EBV infections. In turn, this longer EBV exposure could explain the observed age difference between distinct clinical presentations, with abdominal eBL occurring more often among older children.

Our study has some limitations. First, results are based on case-control data, so temporality of infections and causality of associations cannot be determined. Thus, the observed associations with the two infections could be due to reverse causality since changes in antibody levels could be consequence of eBL disease. The use of hospital oncologic controls might be another limitation. Although we excluded all children diagnosed with hematological cancers, Hodgkin lymphoma or nasopharyngeal tumors, 


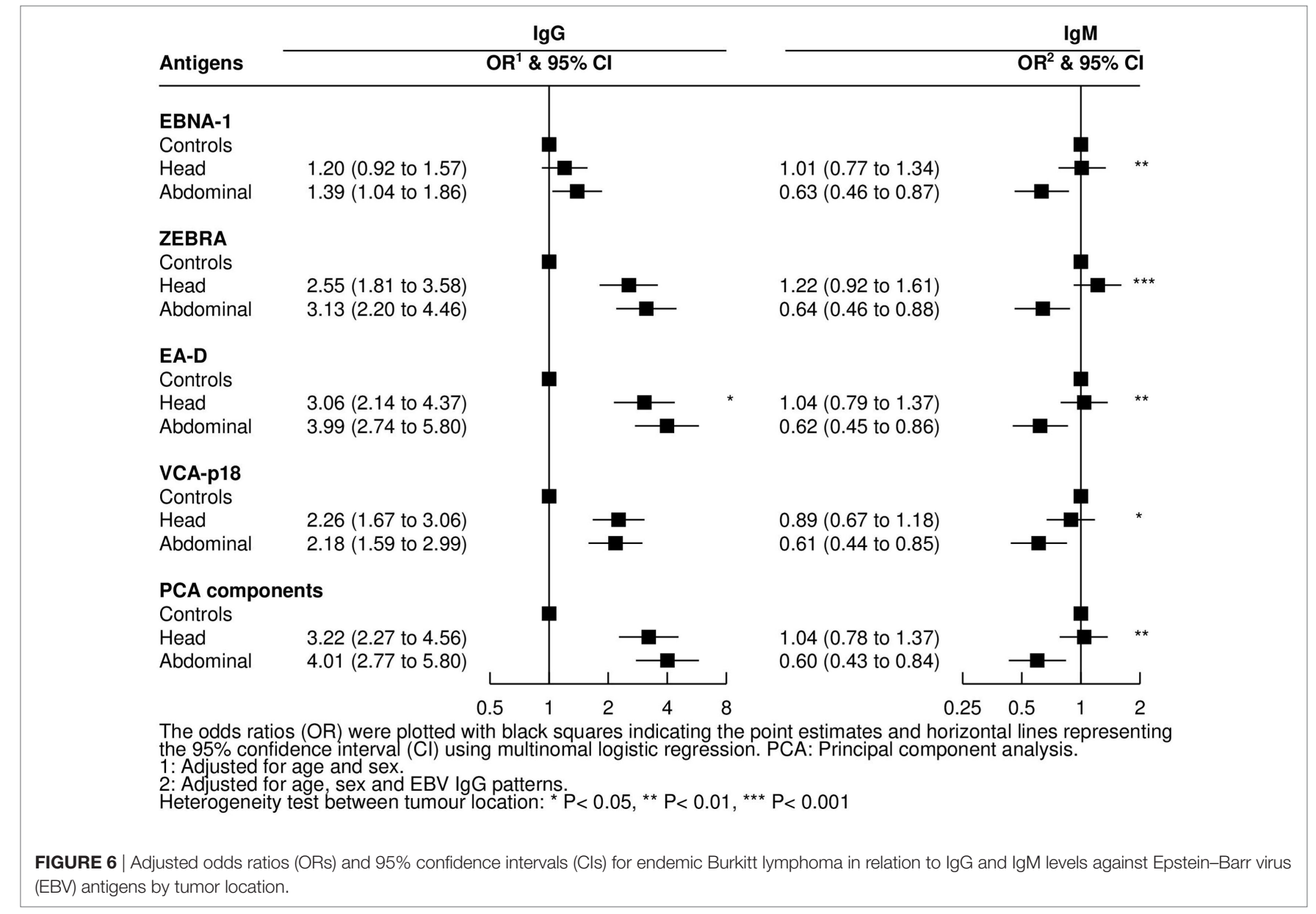

to avoid possible diagnostic overlap with eBL, and to exclude those with known associations with EBV, we cannot discard that some of these other conditions might be affecting the antibody responses to EBV and P. falciparum antigens. Misclassification in cases and tumor location might have occurred, as $25 \%$ of cases and controls did not have a histological verification of diagnosis; children might have had metastasis at hospital arrival, making the classification difficult. In addition, the understanding of the sero-epidemiology of $P$. falciparum is at an early stage, making the interpretation of the findings challenging. It would have been informative to examine the serological responses in relation to EBV and parasite densities, but this information was not available. However, the elevated levels of IgG to EA-D reflect an increased production of EBV DNA and hence might be a surrogate of EBV-viral load. Finally, multiple associations were evaluated and chance in some of them cannot be excluded, thus the weak associations observed for some P. falciparum antigens with eBL and the additive interaction of MSP-3 with EBV must be taken with caution. Nevertheless, the confirmation of previous findings and the internal consistency of results make us confident that the findings are robust.

In conclusion, we found a strong association between EBV and eBL, and identified new associations between specific antibodies to $P$. falciparum, EBV, and eBL. Multiple P. falciparum antibody responses and differential effects by liver- vs blood-stage infections might reflect a complex mechanism whereby the parasite modulates the association between EBV and eBL. Longitudinal studies with more immunological and molecular data on EBV and $P$. falciparum infections are needed to better understand the combined mechanism of the two infections in eBL etiology. Future studies on eBL should collect information on tumor location since the infections might act differently by tumor presentation. The development of vaccines against these two infections would be crucial to protect African children from eBL.

\section{ETHICS STATEMENT}

Ethical approval for the study was obtained from the Oxford Tropical Research Ethics Committee and the Malawian College of Medicine Research and Ethics Committee. The parent or guardian of each child provided written informed consent for their child to be included in the study in accordance with the Declaration of Helsinki.

\section{AUTHORS CONTRIBUTIONS}

Substantial contributions to the conception or design of the work: RA, DC, CO-G, JC, SS, TW, MK, RN, and CD. 
Acquisition of samples/data: NM, RN, EA, SD, DG, CC, VC, AM, and TW. Analysis of samples: RA, MV, JC, and TW. Analysis of data: DC. Interpretation of data: RA, DC, CO-G, SS, TW, MK, RN, and CD. Draft of the manuscript: RA and DC. Critical revision of the draft for important intellectual content, final approval of the version to be published, and agreement to be accountable for all aspects of the work in ensuring that questions related to the accuracy or integrity of any part of the work are appropriately investigated and resolved: all the authors.

\section{ACKNOWLEDGMENTS}

The authors thank the children and their parents/guardians for their participation in the study. The authors are grateful to Professor Elizabeth Molyneux from the Queen Elizabeth Central Hospital in Blantyre for her assistance in the recruitment of children with cancer. From ISGlobal, the authors would like to thank Alfredo Mayor and Aida Valmaseda for antigen supply; Alfons Jimenez for support with the Luminex assay; Laura Puyol for shipment logistics; and Yolanda Benavente for support on $\mathrm{R}$ programming.

\section{REFERENCES}

1. IARC monographs on the evaluation of carcinogenic risks to humans. (2012) 100B. Available from: http://monographs.iarc.fr/ENG/Monographs/vol100B/ index.php

2. Magrath I. The pathogenesis of Burkitt's lymphoma. Adv Cancer Res (1990) 55:133-270. doi:10.1016/S0065-230X(08)60470-4

3. Chattopadhyay PK, Chelimo K, Embury PB, Mulama DH, Sumba PO, Gostick E, et al. Holoendemic malaria exposure is associated with altered Epstein-Barr virus-specific CD8(+) T-cell differentiation. J Virol (2013) 87(3): 1779-88. doi:10.1128/JVI.02158-12

4. Olweny CL. Etiology of endemic Burkitt's lymphoma. IARC Sci Publ (1984) 63:647-53.

5. Simone O, Bejarano MT, Pierce SK, Antonaci S, Wahlgren M, Troye-Blomberg M, et al. TLRs innate immunereceptors and Plasmodium falciparum erythrocyte membrane protein 1 (PfEMP1) CIDRlalpha-driven human polyclonal B-cell activation. Acta Trop (2011) 119(2-3):144-50. doi:10.1016/j.actatropica. 2011.05.005

6. Thorley-Lawson D, Deitsch KW, Duca KA, Torgbor C. The link between Plasmodium falciparum malaria and endemic Burkitt's lymphoma-new insight into a 50-year-old enigma. PLoS Pathog (2016) 12(1):e1005331. doi:10.1371/ journal.ppat.1005331

7. Bouvard V, Baan RA, Grosse Y, Lauby-Secretan B, El Ghissassi F, BenbrahimTallaa L, et al. Carcinogenicity of malaria and of some polyomaviruses. Lancet Oncol (2012) 13(4):339-40. doi:10.1016/S1470-2045(12)70125-0

8. Piriou E, Asito AS, Sumba PO, Fiore N, Middeldorp JM, Moormann AM, et al. Early age at time of primary Epstein-Barr virus infection results in poorly controlled viral infection in infants from Western Kenya: clues to the etiology of endemic Burkitt lymphoma. J Infect Dis (2012) 205(6):906-13. doi:10.1093/ infdis/jir872

9. Reynaldi A, Schlub TE, Chelimo K, Sumba PO, Piriou E, Ogolla S, et al. Impact of Plasmodium falciparum coinfection on longitudinal Epstein-Barr virus kinetics in Kenyan children. J Infect Dis (2016) 213(6):985-91. doi:10.1093/ infdis/jiv525

10. Carpenter LM, Newton R, Casabonne D, Ziegler J, Mbulaiteye S, Mbidde E, et al. Antibodies against malaria and Epstein-Barr virus in childhood Burkitt lymphoma: a case-control study in Uganda. Int J Cancer (2008) 122(6):1319-23. doi:10.1002/ijc. 23254

\section{FUNDING}

This work was supported by the CERCA Institutes Integration Programme [SUMA 2013, promoted and managed by the Secretariat for Universities and Research (SUR) of the Economy and Knowledge Council of the Government of Catalonia, the Agency for Management of University and Research Grants (AGAUR), and the CERCA Institute] and the Instituto de Salud Carlos III, grant number PI14/01422. It also received support from 2014SGR991, 2014SGR756, CIBERESP-groups 36 and 38, PI11/01810, PI14/01219, and Red Temática de Investigación del Cáncer (RD06/0020/0095, RD12/0036/0056), cofounded by FEDER funds/European Regional Development Fund (ERDF). CO-G was recipient of a Sara Borrell-ISCIII fellowship (CD13/00072). The childhood cancer study conducted at the Queen Elizabeth Hospital in Blantyre, Malawi, was supported by Cancer Research UK.

\section{SUPPLEMENTARY MATERIAL}

The Supplementary Material for this article can be found online at http://www.frontiersin.org/article/10.3389/fimmu.2017.01284/ full\#supplementary-material.

11. Mutalima N, Molyneux E, Jaffe H, Kamiza S, Borgstein E, Mkandawire N et al. Associations between Burkitt lymphoma among children in Malawi and infection with HIV, EBV and malaria: results from a case-control study. PLoS One (2008) 3(6):e2505. doi:10.1371/journal.pone.0002505

12. Asito AS, Piriou E, Odada PS, Fiore N, Middeldorp JM, Long C, et al. Elevated anti-Zta IgG levels and EBV viral load are associated with site of tumor presentation in endemic Burkitt's lymphoma patients: a case control study. Infect Agent Cancer (2010) 5:13. doi:10.1186/1750-93785-13

13. Aka P, Vila MC, Jariwala A, Nkrumah F, Emmanuel B, Yagi M, et al. Endemic Burkitt lymphoma is associated with strength and diversity of Plasmodium falciparum malaria stage-specific antigen antibody response. Blood (2013) 122(5):629-35. doi:10.1182/blood-2012-12-475665

14. Fachiroh J, Paramita DK, Hariwiyanto B, Harijadi A, Dahlia HL, Indrasari SR, et al. Single-assay combination of Epstein-Barr virus (EBV) EBNA1- and viral capsid antigen-p18-derived synthetic peptides for measuring anti-EBV immunoglobulin G (IgG) and IgA antibody levels in sera from nasopharyngeal carcinoma patients: options for field screening. J Clin Microbiol (2006) 44(4):1459-67. doi:10.1128/JCM.44.4.1459-1467.2006

15. Waterboer T, Sehr P, Michael KM, Franceschi S, Nieland JD, Joos TO, et al. Multiplex human papillomavirus serology based on in situ-purified glutathione s-transferase fusion proteins. Clin Chem (2005) 51(10):1845-53. doi:10.1373/clinchem.2005.052381

16. Kariu T, Ishino T, Yano K, Chinzei Y, Yuda M. CelTOS, a novel malarial protein that mediates transmission to mosquito and vertebrate hosts. Mol Microbiol (2006) 59(5):1369-79. doi:10.1111/j.1365-2958.2005.05024.x

17. Hillier CJ, Ware LA, Barbosa A, Angov E, Lyon JA, Heppner DG, et al. Process development and analysis of liver-stage antigen 1, a preerythrocyte-stage protein-based vaccine for Plasmodium falciparum. Infect Immun (2005) 73(4):2109-15. doi:10.1128/IAI.73.4.2109-2115.2005

18. Rogers WO, Malik A, Mellouk S, Nakamura K, Rogers MD, Szarfman A, et al. Characterization of Plasmodium falciparum sporozoite surface protein 2. Proc Natl Acad Sci U S A (1992) 89(19):9176-80. doi:10.1073/ pnas.89.19.9176

19. Plassmeyer ML, Reiter K, Shimp RL Jr, Kotova S, Smith PD, Hurt DE, et al. Structure of the Plasmodium falciparum circumsporozoite protein, a leading malaria vaccine candidate. J Biol Chem (2009) 284(39):26951-63. doi:10.1074/ jbc.M109.013706 
20. Kocken CH, Withers-Martinez C, Dubbeld MA, van der Wel A, Hackett F, Valderrama A, et al. High-level expression of the malaria blood-stage vaccine candidate Plasmodium falciparum apical membrane antigen 1 and induction of antibodies that inhibit erythrocyte invasion. Infect Immun (2002) 70(8):4471-6. doi:10.1128/IAI.70.8.4471-4476.2002

21. Pandey KC, Singh S, Pattnaik P, Pillai CR, Pillai U, Lynn A, et al. Bacterially expressed and refolded receptor binding domain of Plasmodium falciparum EBA-175 elicits invasion inhibitory antibodies. Mol Biochem Parasitol (2002) 123(1):23-33. doi:10.1016/S0166-6851(02)00122-6

22. Mayor A, Rovira-Vallbona E, Srivastava A, Sharma SK, Pati SS, Puyol L, et al. Functional and immunological characterization of a Duffy binding-like alpha domain from Plasmodium falciparum erythrocyte membrane protein 1 that mediates rosetting. Infect Immun (2009) 77(9):3857-63. doi:10.1128/ IAI.00049-09

23. Bir N, Yazdani SS, Avril M, Layez C, Gysin J, Chitnis CE. Immunogenicity of Duffy binding-like domains that bind chondroitin sulfate A and protection against pregnancy-associated malaria. Infect Immun (2006) 74(10):5955-63. doi:10.1128/IAI.00481-06

24. Chauhan VS, Yazdani SS, Gaur D. Malaria vaccine development based on merozoite surface proteins of Plasmodium falciparum. Hum Vaccin (2010) 6(9):757-62. doi:10.4161/hv.6.9.12468

25. Siddiqui FA, Dhawan S, Singh S, Singh B, Gupta P, Pandey A, et al. A thrombospondin structural repeat containing rhoptry protein from Plasmodium falciparum mediates erythrocyte invasion. Cell Microbiol (2013) 15(8): 1341-56. doi:10.1111/cmi.12118

26. Sahar T, Reddy KS, Bharadwaj M, Pandey AK, Singh S, Chitnis CE, et al. Plasmodium falciparum reticulocyte binding-like homologue protein 2 (PfRH2) is a key adhesive molecule involved in erythrocyte invasion. PLoS One (2011) 6(2):e17102. doi:10.1371/journal.pone.0017102

27. Reddy KS, Pandey AK, Singh H, Sahar T, Emmanuel A, Chitnis CE, et al. Bacterially expressed full-length recombinant Plasmodium falciparum RH5 protein binds erythrocytes and elicits potent strain-transcending parasite-neutralizing antibodies. Infect Immun (2014) 82(1):152-64. doi:10.1128/ IAI.00970-13

28. Reddy KS, Amlabu E, Pandey AK, Mitra P, Chauhan VS, Gaur D. Multiprotein complex between the GPI-anchored CyRPA with PfRH5 and PfRipr is crucial for Plasmodium falciparum erythrocyte invasion. Proc Natl Acad Sci U S A (2015) 112(4):1179-84. doi:10.1073/pnas.1415466112

29. Dutta S, Lee SY, Batchelor AH, Lanar DE. Structural basis of antigenic escape of a malaria vaccine candidate. Proc Natl Acad Sci U S A (2007) 104(30):12488-93. doi:10.1073/pnas.0701464104

30. Angov E, Aufiero BM, Turgeon AM, Van Handenhove M, Ockenhouse CF, Kester KE, et al. Development and pre-clinical analysis of a Plasmodium falciparum merozoite surface protein-1(42) malaria vaccine. Mol Biochem Parasitol (2003) 128(2):195-204. doi:10.1016/S0166-6851(03)00077-X

31. Campo JJ, Dobano C, Sacarlal J, Guinovart C, Mayor A, Angov E, et al. Impact of the RTS,S malaria vaccine candidate on naturally acquired antibody responses to multiple asexual blood stage antigens. PLoS One (2011) 6(10):e25779. doi:10.1371/journal.pone.0025779

32. Karachaliou M, Chatzi L, Roumeliotaki T, Kampouri M, Kyriklaki A, Koutra K, et al. Common infections with polyomaviruses and herpesviruses and neuropsychological development at 4 years of age, the Rhea birth cohort in Crete, Greece. J Child Psychol Psychiatry (2016) 57:1268-76. doi:10.1111/ jcpp. 12582

33. Morrison BJ, Labo N, Miley WJ, Whitby D. Serodiagnosis for tumor viruses. Semin Oncol (2015) 42(2):191-206. doi:10.1053/j.seminoncol.2014.12.024

34. Altman DG, Royston P. The cost of dichotomising continuous variables. $B M J$ (2006) 332(7549):1080. doi:10.1136/bmj.332.7549.1080

35. StataCorp. Stata: Release 13. Statistical Software. College Station, TX: StataCorp LP (2013).

36. R Development Core Team. R: A Language and Environment for Statistical Computing. Vienna, Austria: R Foundation for Statistical Computing (2008). Available from: http://www.R-project.org

37. Friedman J, Hastie T, Tibshirani R. Regularization paths for generalized linear models via coordinate descent. J Stat Softw (2010) 33(1):1-22. doi:10.18637/ jss.v033.i01
38. Sill M, Hielscher T, Becker N, Zucknick M. Extended inference with lasso and elastic-net regularized Cox and generalized linear models. J Stat Softw (2014) 62(5):1-22. doi:10.18637/jss.v062.i05

39. Gaujoux R, Seoighe C. A flexible R package for nonnegative matrix factorization. BMC Bioinformatics (2010) 11:367. doi:10.1186/1471-2105-11-367

40. Reynaldi A, Schlub TE, Piriou E, Ogolla S, Sumba OP, Moormann AM, et al. Modeling of EBV infection and antibody responses in Kenyan infants with different levels of malaria exposure shows maternal antibody decay is a major determinant of early EBV infection. J Infect Dis (2016) 214:1390-8. doi:10.1093/infdis/jiw396

41. Geser A, de The G, Lenoir G, Day NE, Williams EH. Final case reporting from the Ugandan prospective study of the relationship between EBV and Burkitt's lymphoma. Int J Cancer (1982) 29(4):397-400. doi:10.1002/ ijc. 2910290406

42. Doolan DL, Dobano C, Baird JK. Acquired immunity to malaria. Clin Microbiol Rev (2009) 22(1):13-36, Table of Contents. doi:10.1128/CMR.00025-08

43. Beeson JG, Drew DR, Boyle MJ, Feng G, Fowkes FJ, Richards JS. Merozoite surface proteins in red blood cell invasion, immunity and vaccines against malaria. FEMS Microbiol Rev (2016) 40(3):343-72. doi:10.1093/femsre/ fuw001

44. Soe S, Theisen M, Roussilhon C, Aye KS, Druilhe P. Association between protection against clinical malaria and antibodies to merozoite surface antigens in an area of hyperendemicity in Myanmar: complementarity between responses to merozoite surface protein 3 and the 220-kilodalton glutamate-rich protein. Infect Immun (2004) 72(1):247-52. doi:10.1128/IAI.72.1.247-252.2004

45. Roussilhon C, Oeuvray C, Muller-Graf C, Tall A, Rogier C, Trape JF, et al. Long-term clinical protection from falciparum malaria is strongly associated with IgG3 antibodies to merozoite surface protein 3. PLoS Med (2007) 4(11):e320. doi:10.1371/journal.pmed.0040320

46. Dodoo D, Atuguba F, Bosomprah S, Ansah NA, Ansah P, Lamptey H, et al. Antibody levels to multiple malaria vaccine candidate antigens in relation to clinical malaria episodes in children in the Kassena-Nankana district of Northern Ghana. Malar J (2011) 10:108. doi:10.1186/1475-2875-10-108

47. Kangoye DT, Nebie I, Yaro JB, Debe S, Traore S, Ouedraogo O, et al. Plasmodium falciparum malaria in children aged $0-2$ years: the role of foetal haemoglobin and maternal antibodies to two asexual malaria vaccine candidates (MSP3 and GLURP). PLoS One (2014) 9(9):e107965. doi:10.1371/ journal.pone. 0107965

48. Aguiar JC, Bolton J, Wanga J, Sacci JB, Iriko H, Mazeika JK, et al. Discovery of novel Plasmodium falciparum pre-erythrocytic antigens for vaccine development. PLoS One (2015) 10(8):e0136109. doi:10.1371/journal. pone. 0136109

49. Kanoi BN, Takashima E, Morita M, White MT, Palacpac NM, Ntege EH, et al. Antibody profiles to wheat germ cell-free system synthesized Plasmodium falciparum proteins correlate with protection from symptomatic malaria in Uganda. Vaccine (2017) 35(6):873-81. doi:10.1016/j.vaccine.2017.01.001

50. Espinosa DA, Vega-Rodriguez J, Flores-Garcia Y, Noe AR, Munoz C, Coleman R, et al. The Plasmodium falciparum cell-traversal protein for ookinetes and sporozoites as a candidate for preerythrocytic and transmission-blocking vaccines. Infect Immun (2017) 85(2):e498-416. doi:10.1128/IAI. 00498-16

Conflict of Interest Statement: The authors declare that the research was conducted in the absence of any commercial or financial relationships that could be construed as a potential conflict of interest.

The reviewer KF and handling editor declared their shared affiliation.

Copyright (c) 2017 Aguilar, Casabonne, O’Callaghan-Gordo, Vidal, Campo, Mutalima, Angov, Dutta, Gaur, Chitnis, Chauhan, Michel, de Sanjosé, Waterboer, Kogevinas, Newton and Dobaño. This is an open-access article distributed under the terms of the Creative Commons Attribution License (CC BY). The use, distribution or reproduction in other forums is permitted, provided the original author(s) or licensor are credited and that the original publication in this journal is cited, in accordance with accepted academic practice. No use, distribution or reproduction is permitted which does not comply with these terms. 